\title{
EL CINE DOCUMENTAL MEXICANO HECHO POR MUJERES
}

\section{Mexican documentary film made by women}

\author{
María del Carmen DE LARA RANGEL \\ Profesora titular. Ciudad de México, México \\ Universidad Nacional Autónoma de México Centro Universitario de Estudios Cinematográficos \\ E-mail: direcuec@gmail.com
}

(iD) ORCID

Fecha de recepción del artículo: 31/03/2019

Fecha de aceptación definitiva: 17/04/2019

\section{RESUMEN}

La construcción cultural y la aportación de las mujeres, sobre todo en el ámbito documental, pareciera ser escondida por una visión patriarcal. La historia de las mujeres en el cine documental mexicano ha sido marcada por la exclusión, la falta de tolerancia y algunas de las historias de estas creadoras en México han sido rescatadas por una de las historiadoras más dedicadas a temas vinculados con el quehacer cinematográfico: Patricia Torres San Martin.

Hoy día apenas se empiezan a rescatar las labores y sinsabores de mujeres como Elena Valenzuela, otra documentalista que en su momento no sólo filmó varios cortometrajes, sino que fue una de las pioneras en la creación de Archivos Fílmicos y que descubrimos gracias al trabajo de esta investigadora.

El valor del papel de las mujeres documentalistas mexicanas comienza a tomar forma y a plantearse en el medio hoy día gracias a los movimientos feministas que con dispositivos como los medios digitales, distribuyen su voz en todos los continentes.

Debido a que el cine es parte del conocimiento y la formación en las sociedades actuales, esto nos lleva a la necesidad de sistematizar cada vez más las memorias e historias de quienes fueron pioneras en los distintos ámbitos.

Palabras clave: cine feminista; cine mexicano; cine mujeres.

\begin{abstract}
Cultural construction and women's efforts in documentary films seem to be hidden by a patriarchal view. Women's history in Mexican documentary films has been excluded by the lack of tolerance, and some of these women in Mexico have been rescued by one of the most dedicated women on cinematography topics: Patricia Torres San Martin.

Nowadays the work and history of different women in cinematography and documentary film are being rescued thanks to the work of Patricia Torres. One example is Elena Valenzuela, film director, a pioneer on film archives in México during the first part of the $20^{\text {th }}$ century.

The value of women in documentary films in Mexico takes form and begins to set up on digital media today thanks to all the feminist movements that are socialized on different devices which distribute their voice across all continents.
\end{abstract}


Because cinematography is a very important part of knowledge for society, there is a need to systematize the different memories of all pioneering women in this art.

Key words: feminist cinematography; mexican cinematography; women in cinematography.

\section{INTRODUCCIÓN}

Tuvieron que pasar más de 100 años de historia para descubrir las aportaciones que las mujeres han hecho al cine. En años recientes, gracias a Marquise Lepage, realizadora originaria de Quebec, pudimos ver las pequeñas ficciones de Alice Guy Blanchet y descubrir así que antes de Méliès, una mujer trabajadora de Gaumont había sido prácticamente ignorada de la historia del Cine Mundial (Solange Collin, 1996).

Las hermanas Adriana y Dolores Elhers fueron pioneras en el documental pero fue tal vez por casualidad y por la decisión de traer el cinematógrafo a México, situación que llevó a los Hermanos Alba y Toscano a formar uno de los archivos más importantes de la Revolución Mexicana.

Las hermanas Adriana y Dolores Elhers fueron pioneras de la incipiente cinematografía mexicana: se formaron en el oficio gracias al apoyo financiero del presidente Venustiano Carranza, filmaron documentales, procesaron películas, vendieron aparatos de proyección y trabajaron para la primera oficina de censura que hubo en México. Entre 1922 y 1929, realizaron el noticiero semanal La Revista Elhers, que mostraba los acontecimientos del momento -catástrofes, desfiles, manifestaciones- y que ellas vendían directamente a los exhibidores. El inicio del cine sonoro produjo muchos cambios, pero no alteró la situación de las mujeres. En general, permanecían frente a la cámara y no detrás de ella (Martín, 2008, p. 113).

Después de las Elhers, la actriz del primer Silente de Santa, la joven entusiasta Elena Sánchez Valenzuela, según las investigaciones de Patricia Torres, definen la lucha en contra de los relatos históricos que se encargaron de minimizar el trabajo de mujeres precursoras de nuestro Cine Nacional, su participación, es un espacio que ha marcado tanto en lo simbólico como en lo real los productos de la cinematografía en México (Martín, 2008).

Re significar y tener una memoria de la historia y la aportación de las mujeres en el caso del Cine Nacional nos lleva a analizar, como premisa fundamental de esta obra, cómo se trataban y se contaban las historias en el cine de ficción y que empieza a dictar pautas sobre el tratamiento de los personajes femeninos, es a partir de la consideración de las mujeres como objetos u adornos, su cosificación, permitió que en la mayor parte de los casos se invisibilizaran. Su labor, como intersticio que enfrenta al pasado formativo con el presente realizador de cientos de mujeres que proponen con su lente una mirada crítica y definida sobre su representación como género en la Cinematografía y la subjetividad colectiva de audiencias formadas desde la hegemonía visual de los mercados comerciales. 


\subsection{El cine en masculino, una resistencia bistórica desde nuestra experiencia como documentalistas}

La mujer exhibida como objeto sexual es el leitmotiv del espectáculo visual en el cine dominante: ella es observada y significa el deseo. Pero a pesar de su objetivación sigue evocando la amenaza de la castración, por lo que se emplean dos estrategias para neutralizarla: convertirla en fetiche (fetichismo) o tratar de resolver su misterio para finalmente devaluarla, castigarla o redimirla (sadismo). Estos mecanismos, que responden a necesidades patriarcales, son invisibilizados mediante la manipulación de los códigos cinematográficos (Sandoval, 2013, p. 10).

La representación de la mujer como fetiche, su vulnerabilidad expuesta para acercar a un espectador en masculino que otorga un veredicto sobre el final (casi siempre trágico) propuso espacios de resistencia en aquellas mujeres que se enfrentaron con el ingenio cinematográfico, un espacio político avasallado por las estructuras patriarcales de los contenidos y las formas de representación del Cine Clásico Hollywoodense, éstas crearon un inconsciente colectivo, donde el espectador identificado por la naturalización de una diferencia sexual y su mirada desde el erotismo crearon en la subjetividad colectiva, mecanismos de fascinación sobre el cine y el papel de la mujer en él (Mulvey, 1975).

Elena Sánchez Valenzuela se negó a continuar por esta vía, en cambio comenzó a desarrollar un trabajo de rescate de imágenes, entendiendo el poder de la imagen como expresión que podría plasmarse de otra manera. Es la segunda documentalista que trabaja para la Secretaría de Educación Pública del gobierno de México. A Elena le encomiendan realizar la coordinación del documental Michoacán, pero también con ella al frente de un departamento de la SEP durante el Vasconcelismo, descubrimos su propuesta visionaria de crear Filmotecas, lo que la lleva al viaje en esa época por Latinoamérica y Centroamérica, especialmente a Guatemala para proponer y establecer una red por los países visitados. Para Patricia Torres San Martín, la parte fascinante de esta trayectoria, se encuentra después de haber participado como actriz donde se asientan muchos de los estereotipos que seguirá desarrollando el cine mexicano por varias décadas.

Entre estas pioneras y el cine documental feminista pasaron casi 40 años y se desconoce si existieron otras producciones de cine realizadas por mujeres en el género documental.

En México nació el Colectivo Cine Mujer (1975-1987), la creadora de esta idea a partir de la producción fílmica, fue en ese entonces la estudiante de cine Rosa Martha Fernández, quien inicio un análisis desde otra perspectiva, al hablar de las protestas con una amplia gama de exigencias centradas en la voluntad de abordar un cine propiamente femenino, emancipador y político. Rosa Martha se forma en el pensamiento feminista con libros de Simone de Beauvoir como el Segundo Sexo y con el texto revelador de esta generación de Germaine Greer: "Lo personal sigue siendo político. La feminista del nuevo milenio no puede dejar de ser consciente de que la opresión se ejerce en y a través de sus relaciones más íntimas, empezando por la más íntima de todas: la relación con el propio cuerpo» (Amoros, 1985).

En este contexto con la formación de los pequeños grupos que empezaban a reconocerse como personas, sujetos de sus historias y junto con la formación universitaria post movimiento de 1968, la visión crítica y la demanda de inclusión permitió que se formara un grupo donde la mayoría de los filmes realizados en $16 \mathrm{~mm}$ fueran 
dirigidos por alumnas del Centro Universitario de Estudios Cinematográficos de la Universidad Nacional Autónoma de México (UNAM) y que tuvieron el gran mérito de abordar temas tabú del momento. En el documental Cosas de mujeres, 1975-1978, de Rosa Martha Fernández, propone la mezcla del documental y la ficción, su narrativa desarrolla la historia de lo personal y lo político, premisa básica del feminismo de la época. La historia nos habla de la importancia de permitir decisiones sobre el cuerpo, la maternidad elegida y la necesidad de servicios médicos legales.

Rosa Martha se reúne con mujeres que migran a Latinoamérica procedentes de Europa y EU, en búsqueda de las raíces de ese mundo de profundos contrastes sociales, que a partir también de la influencia de los movimientos sociales de países como Cuba y Colombia, se interesan en otra forma de construirse, jóvenes en busca de panoramas nuevos y equitativos de la todavía muy distante izquierda machista que es incapaz de incorporar estas propuestas y que no ve más que la lucha de clases desde un punto de vista de la política y no de la revolución interna que significa el cuestionamiento de la familia y la reproducción como sentido de «ser mujer».

También el Colectivo Cine Mujer representa el trabajo doméstico con el cortometraje Vicios en la cocina, 1977, de Beatriz Mira, basada en un poema de Sylvia Plath. Este proyecto fílmico, habla del tedio, de la repetición, mientras escuchamos como contrapunto la canción de "Dos Gardenias para ti», en la voz de Bienvenido Granda. Este documental registra las labores domésticas y mezclado con el poema que le da la profundidad de la mirada en torno a la doble jornada, una crítica desde la experiencia femenina.

Después de estos dos ejercicios narrativos, Rosa Martha llama a un grupo de interesadas feministas estudiantes del CUEC a discutir en colectivo la siguiente realización sobre el tema de la violación con Rompiendo el silencio en 1979. Uniendo así las distintas inquietudes de Ana Victoria Jiménez, fotógrafa que había ya documentando en esa época las expresiones de un movimiento desde las protestas lideradas por Marta Acevedo, recién llegada de San Francisco, California, empapada de las expresiones que se dan en Estados Unidos en torno a la liberación femenina, lectora de textos como los de Kate Miller, como el de salud publicado por el Colectivo de Mujeres de Boston llamado "Nuestros cuerpos, nuestras vidas".

Rosa Martha hace un llamado a Helen Camus, Sybil Hayem, Laura Rosseti Mónica Meyer, Lilian Liberman y algunas otras mujeres que no son estudiantes del CUEC, pero que son feministas, llama a la reflexión, a la discusión para realizar ejercicios fílmicos y tratar de armar un guión, donde se narre desde diversos puntos de vista, donde también la improvisación y el pensamiento crítico feminista construya un grito colectivo desesperado de las mujeres y la violación.

Rosa Martha también se pregunta: ¿Qué pasa con los hombres?, lo hace con su producción documental al entrevistar a un preso acusado de violación, trata de oponerse y provocar la ruptura de los cánones que de acuerdo a una sociedad de doble moral que no habla de estas problemáticas, intenta fortalecer la libre expresión y empapada por el contexto de la época que algunos consideraban como base de un feminismo radical, originados en los movimientos contestatarios de los años 60 del siglo XX.

Se asume así en esos años, casi como categoría social y política, la diferencia en la relación de poder entre hombres y mujeres, se traduce en desigualdad, después inequidad, más tarde en la desgastada y poco entendida visión de género que incluye a comunidades más diversas y que pasa al plano de lo político, pero el viejo lema de 
lo personal es político, sigue hasta el Metoo siendo una demanda que las feminismos tienen hasta la fecha.

Por supuesto la prostitución es uno de los temas que genera una visión de desigualdad y de cuerpos mayormente femeninos pero diversos, que son estigmatizados por la sociedad y que en el Cine Nacional Mexicano ocupan una serie de versiones en los años 70's y 80's, de ahí surge No es por gusto, 1981, de Maricarmen de Lara -autora del texto- y María Eugenia Tamez. No es por gusto, inicia con una investigación seria e importante, donde participamos junto con Alfonso Morales, con una idea vaga de la metodología documental que nos hacía aferrarnos al cine directo como tratamiento en la mayor parte de la película, es un claro ejemplo de realismo subversivo, pues decidimos abarcar tres espacios, la vida cotidiana que contradice el estereotipo de prostituta, la mujer madre, amiga y que a la vez critica el recurrente en el cine mexicano producido entre los años 50, 60 y 70, el espacio de trabajo, la vida pública, la violencia y el riesgo. Lo más complicado de abordar en esa época de equipos cinematográficos grandes y difíciles de esconder durante la filmación en cine con magazines de 10 minutos y complicada sensibilidad de película, nos llevaba también a romper nuestros prejuicios como realizadoras.

Nosotras pensábamos que los padrotes eran unos señores malos de gazné como las películas de Mauricio Garcés, resultando que en casa de las protagonistas ellos nos ofrecían té y se ponían a cuidar a los niños, trían la comida mientras realizábamos las entrevistas. Nos dimos cuenta, que en muchos casos, existía una relación amorosa, como la que tenímos cualquiera. Y entonces, como que hay muchos mitos que se te van quitando (De Lara Rangel, 1981).

El espacio carcelario fue donde se realizó la investigación de campo, ahí llevando un pequeño cine club pudimos compartir tiempo y angustia de nuestras protagonistas, conversar, relacionarnos y tratar de convencerlas de aparecer en la imagen, pero ya en nosotras con una fuerte mirada feminista, critica del poder, contra la desigualdad. Decidimos contar las historias de quienes todavía se autonombraban prostitutas y no trabajadoras sexuales, ver que ellas eran penalizadas y reprimidas, mientras el cliente podía mirar como ellas eran detenidas y ellos no. Fue una experiencia que hasta la fecha nos lleva a ver el enorme rezago social que han padecido las mujeres que se dedican a la prostitución.

Posteriormente a la participación en el Colectivo Cine Mujer se afianza un compromiso con el feminismo que marcará todo el trabajo y visión de la vida de las mujeres que en él participaron, un activismo político debido que: nosotras empezamos a tratar problemáticas que los hombres no trataban porque no les interesaban, porque no les afectaban.

Tanto el aborto como la violación, el trabajo sexual, el trabajo doméstico, fueron temas que empezamos a tratar las mujeres documentalistas, no nada más en México sino en el mundo, Chantal Akermann y Agnes Varda hablaban de la vida cotidiana.

Nos interesaba y actualmente como mujeres nos interesa, visibilizar las historias de mujeres. Ser feminista ha significado asumir una postura política que comienza por la denuncia de las opresiones impuestas a las mujeres en una sociedad patriarcal. Ese es siempre su punto de vista y su conocimiento situado, que no depende de una esencia inherente al cuerpo sexuado femenino, sino del desarrollo de una conciencia de la desigualdad de género que opera junto con la violencia del capitalismo salvaje. 
Vida de Ángel fue uno de los ejercicios colectivos un corto del encuentro realizado por mujeres campesinas un fin de semana en la ciudad de México y uno de lo últimos de una etapa del colectvo como tal.

No es la corporeidad la que te hace feminista, hay hombres feministas y mujeres machistas. Esta pluralidad del pensamiento feminista también transita en la realización del documental, donde el problema general no es el hombre, sino el machismo y una sociedad patriarcal que lo avala, y todo aquello que nos convierte en mercancías a mujeres y hombres en la sociedad contemporánea, de alguna manera contribuye al sometimiento de las mujeres en primera instancia. Y en ese sometimiento donde se encuentra violencia contra las mujeres, que forma parte también de la expresión y la resistencia de las documentalistas en sus películas.

La colectividad se crea y la visión que tenemos en ese momento, motivada por el contexto político del inicial activismo, es la de contar historias otorgando un especial énfasis a la desigualdad social, es así como siete mujeres en la época de vías terrestres no asfaltadas, deciden viajar a una comunidad, bajo la dirección de Sonia Fritz para hacer una película en torno al Indigenismo Femenino: Yalaltecas, este documental narra también la vida cotidiana de mujeres de la sierra de Oaxaca que deciden exigir en su comunidad el servicio de guardería durante la década de los ochenta, prematura y visionaria propuesta de Sofía Robles, una de las mujeres indígenas de mayor liderazgo en su comunidad, nos lleva a una aventura de la llamada segunda etapa del colectivo, esta vez como película independiente partimos en una camioneta por un viaje de filmación en $16 \mathrm{~mm}$, las entonces nuevas integrantes, importancia que tuvo en la mirada y la integración de Guadalupe Sánchez, Maripi Sáenz, Laura Ruiz, Penélope Simpson, Ángeles Necoechea, Sonia Fritz y Maricarmen De Lara, nos permitirá adentrarnos en el México de la más dura condición femenina y a la vez de una de las culturas más importantes y profundas, la cultura Zapoteca, y su relación con otras culturas de la zona como la Mixe.

Emplear el Cine documental como crítica y reflexión, herramienta de debate de ideas, de difusión de usos y costumbres anquilosados y pertenecientes a la cultura dominada por la visión heterosexuada y patriarcal, nos abrió horizonte para desarrollar una producción más como Colectivo Cine Mujer, Milagros y remedios, de Ángeles Necoechea, estudiante del CCC. La vida y el trabajo de un grupo de mujeres en la ciudad de México en uno de los barrios populares denominado colonia Morelos y la subsistencia de los oficios paralelos como: la costura, inyecciones o venta de golosinas y alimentos callejeros, llamados "milagros» de manutención para mujeres que no tendrían acceso a la educación media y que tenían familias dependientes de esos ingresos. El difícil periplo para subsanar la falta de oportunidad, las ingeniosas maneras de defender un espacio donde se habita, Milagros y remedios de Colectiva Hilván nos habla una vez más de esta desigualdad social.

Durante 1985, la mayoría de nosotras, egresadas de las escuelas de Cine, comenzamos con una búsqueda individual de la construcción de historias que nos definirían en la producción fílmica y sobre todo como realizadoras mexicanas. Para el Colectivo la propuesta también desarrolló una ficción, Bordando la Frontera, dirigida por la misma Ángeles, historia de las maquiladoras en Ciudad Juárez.

Fue un momento emblemático, productivo. De ahí un terremoto devastador produjo que varias mujeres salieran a documentar el hecho, algunas con sus cámaras fotográficas, otras de forma colectiva debido a los pocos insumos fílmicos que encontraban entre amigos y distribuidores. Esta catástrofe nos llevó a realizar No les 
pedimos un viaje a la luna, donde las mujeres trabajadoras de la costura, que vivían en condiciones laborales ilegales y las cuales frente a la caída de los edificios donde trabajaban y por consecuencia de la misma corrupción Estado-empresarial, muchas de sus compañeras quedaron atrapadas y fallecieron entre los escombros. Estas mujeres sobrevivientes, abrieron su vida para luchar por un Sindicato Independiente: 19 de septiembre. El documental narra las condiciones límite que llevan a estas mujeres obreras a la lucha en la calle por la reivindicación de sus derechos laborales, y representa el proceso de tomar conciencia de forma colectiva sobre las realidades que no sólo trabajadoras de la costura si no de cientos de obreros condicionados por las necesidades de sobrevivir en una ciudad monstro como la Ciudad de México enfrentaron durante un evento de tal naturaleza. Ahí estábamos todos los mexicanos, reflejados con nuestra realidad, y un Estado donde sus instituciones omitieron los derechos más básicos del ser humano.

Tres hitos entonces marcan nuestro cine y llevan a siguientes generaciones, varios años después, a fortalecer el discurso cinematográfico del documental femenino: La fusión entre lo político, el cine, la narración y la importancia de la mirada, empleando la herramienta audiovisual como manera de abrir espacios de discusión.

Durante varias décadas, estas películas tuvieron escasa difusión fuera de los circuitos universitarios y políticos, no consiguieron una exposición comercial sino hasta años posteriores. La escasez de información sobre estas producciones nos llevó hacia estos circuitos para abrir espacios. Para el documental, los formatos de video permitieron una mayor producción, en muchos casos de forma independiente. Como realizadoras en muchos de los casos, optamos por este formato y nos vinculamos a Organizaciones de la Sociedad Civil, huyendo de la censura y limitación para realizar proyectos, que ya se construían con el ideal del Bienestar de la Cultura a partir de la concentración de becarios artistas y realizadores con las instituciones culturales y unos años después con el Tratado de Libre Comercio de América del Norte, que sometió al cine nacional entregando mediante la legislación, a las grandes empresas norteamericanas los espacios en pantalla a nivel nacional.

Este proceso personal de lucha por los espacios donde los temas de las mujeres fueran parte integral de una reeducación en México, me permitió la realización de varios documentales en torno a los derechos de las mujeres, como: Quizás...el saber más (1993), Nosotras también: Mujer y SIDA en México (1994), Decisiones Difíciles (1995), Estamos rodeados de tentaciones (1996), No sólo es la sustancia (2003), Paulina en el Nombre de la Ley (2000-04), Boxeadoras mexicanas, El difícil oficio de los puñetazos (2002), Serie Género e Identidades Sexuales (2000), La vela de las Auténticas Intrépidas Buscadoras del Peligro (1999-2000), Mitos y Mitotes del Falso Matriarcado Juchiteco, Voces Silenciadas, Libertad Amenazada (2008), ¿Más Vale maña que fuerza? (2007), Reflexiones Ciudadanas (2009) Expedientes (contra la criminalización de la interrupción del embarazo) (2011) Alaide Foppa, La Sin Ventura (2014), entre otras.

Como parte de una generación de mujeres que entendieron la importancia de expresar tópicos inherentes a las mujeres, sus espacios de vida, su subjetividad, completamente alejada de la mirada hegemónica sexuada y castigada de la cinematografía mundial, estos colectivos de mujeres cineastas se potenció con aquellos que nacieron en todo el mundo, y que representaron las problemáticas de vida de sus contextos. No estuvimos solas, nos acompañamos buscando siempre con los proveedores de películas, las bibliotecas y las amistades viajeras esas películas que construían una educación amplia de formas y miradas del quehacer cinematográfico feminista. 


\subsection{Mujeres Documentalistas, miradas feministas que trascienden en la enseñanza actual de la cinematografía}

Entre otras expresiones con la llegada del video y continuando una línea de formación en las escuelas, varias mujeres tuvieron acceso a la narrativa documental, la perspectiva feminista y de denuncia en la pantalla cinematográfica. Estas expresiones, así como en los inicios de los colectivos de Cine Mujer, se traducen en referencias actuales para una formación crítica, de los espacios feministas de jóvenes realizadoras que hoy día con las ventanas digitales, exponen temas de mayor diversidad de género, derechos humanos e intereses naturales desde lo femenino, donde la explotación de los cuerpos es cuestionada en pequeños cine minutos y que proponen la ampliación de nuestras miradas.

Grandes ejemplos se produjeron durante la década de los noventa como en el caso de María Inés Roque y Guadalupe Miranda con el levantamiento zapatista del 1 de enero del '94, donde filman en Chiapas el documental Las Compañeras Tienen Grado (1995), en este documental refleja la participación política de las mujeres durante uno de los movimientos sociales más importantes del México que tenía sin representación alguna las problemáticas de las mujeres indígenas y que nos presenta la importante participación y propuesta de los liderazgos de la organización y sus cuestionamientos al machismo.

María Inés (Argenmex), estudió en la Universidad Autónoma Metropolitana y realiza más tarde otro documental, donde se pregunta sobre su padre, Julio Iván Roqué, "Lino", fundador de FAR en Córdoba, quien luego integró la conducción de Montoneros en 1977 (año en el que ella migra a México) y que aún permanece desaparecido. Con el documental Papá Iván (2000) explora a partir de cartas, su historia que es la historia de muchos de los Argentinos que salen muy jóvenes huyendo de la dictadura militar y se terminan de formar como cineastas en México.

A partir del llamado de atención por parte de organizaciones civiles e investigadoras sobre los casos de Feminicidio en todo el país desde la frontera de Ciudad Juárez Chihuahua, las desapariciones y asesinatos de mujeres representaron un tema importante que debía cuestionar al Estado y los flujos económicos del Neoliberalismo que se estructuraba en el país. Lourdes Portillo documentalista Chicana, realiza el documental Señorita Extraviada (2001), utilizando una estructura de investigación a partir de lo publicado por Fuentes Berain y posteriormente el libro de Sergio González Rodríguez Huesos en el Desierto. Lourdes se traslada con un equipo de fotógrafo y sonidista norteamericanos a Ciudad Juárez Chihuahua, para tratar de visibilizar el horror de las desaparecidas y asesinadas en esa Ciudad fronteriza.

La película contada por Lourdes en voz en off, intenta comprender lo que sucede, trata de explicar lo inexplicable: la ola de crímenes brutales contra jóvenes en su mayoría adolescentes, trabajadoras de la maquila. Los bárbaros asesinatos en serie cometidos en esa zona del país y repetidos año tras año, incluyendo niñas violadas, torturadas, cuyos cadáveres son arrojados al desierto, las huellas de múltiples asesinatos de mujeres que no dejan explicación alguna y la complicidad policiaca. Este primer documental sobre el tema, desata algunas reacciones de la opinión pública norteamericana y lleva a varias reconocidas actrices como Jane Fonda a alzar la voz contra las autoridades mexicanas, que ocultan, simulan y no resuelven.

Surgen también documentales testimoniales en el 2004 sobre las mujeres presas en Puente Grande, Jalisco, Relatos desde el encierro de Guadalupe Miranda. Mujeres que 
dan su testimonio de vida, de su soledad y de la experiencia emocional del encierro carcelario. Desde su intimidad reflexionan sobre la condición humana, la sobrevivencia y el concepto de libertad, también nos hablan de la profunda desigualdad e inequidad, la situación que las coloca tras las rejas. La trampa abusiva que las lleva a ser utilizadas para trasladar droga y la maternidad en la cárcel.

Durante los años subsecuentes aparecieron potentes discursos documentales, la propuesta digital y la facilidad de nuevas tecnologías promovió aún más la ondanada de expresiones de mujeres en el documental, ligadas a los movimientos sociales. La conversación se pudo desarrollar de mejor manera con estos cambios, el sonido y los avances permitieron más nitidez y mejor empleo dramático del mismo en la dramaturgia y la construcción de los relatos.

Los Festivales de documental empezaron a tomar en cuenta la continuación de los primeros caminos que marcaron a un cine feminista determinado en representar a las mujeres. Los feminismos interviniendo los modos de representación en el documental, el cine observacional mezclado con la necesidad de un público ávido por ver reflejado en las pantallas los sucesos y los lenguajes con una propuesta de análisis y visión muy lejana a la de las mujeres objetualizadas en las películas comerciales.

Las intervenciones feministas están presentes en los documentales subsecuentes, la producción se amplificó reconociéndose, sólo en algunos casos como feminista, pero continuando el desarrollo de una manera de hablar como sujetos de las historias desarrollado una prolífica cantidad de producciones e intercambios con el público y estableciendo vínculos entre sus experiencias como sujetos de género y su obra cinematográfica.

Destaco algunas producciones de mujeres que abrieron espacios de reconocimiento en el documental contemporáneo, donde los temas que representan en pantalla, abren las realidades de la construcción del patriarcado violento hacia los cuerpos cosificados mediáticamente.

Christiane Burkhard con Trazando Aleida (2008) abre el tema de la violencia del Estado sobre la disidencia durante la Guerra Sucia en México en los años setenta. Tema oculto en el Archivo General de la Nación por décadas llega a la pantalla con la historia de Aleida, quien justamente y a partir de la apertura del archivo durante el año 2000, inicia la búsqueda de su familia. Dos hermanos que fueron separados por la represión del Estado hacia los disidentes guerrilleros, proponen una mirada íntima, pero que refleja nuestra historia de todos nosotros, es una mirada al pasado y el presente de México, el acompañamiento con la cámara al momento crucial de la vida de la protagonista y el reencuentro con el pasado familiar, oculto por décadas violentando la infancia de la protagonista.

Lucia Gajá incursiona también en la temática de la desigualdad migrante con $M i$ vida Adentro (2009), filmada en Austin Texas, donde nos habla de la situación de racismo y desigualdad de una mujer mexicana que padece una doble injusticia al ser tratada de manera humillante como ilegal y al ser enfrentada al sistema de justicia norteamericano, acusada de un crimen que no cometió. Este relato filmado como una Road Movie, reflexiona en torno a la vulnerabilidad y nos hace ver como el sistema judicial norteamericano victimiza a las migrantes, el choque cultural frente a la exclusión. Más tarde esta realizadora realiza Batallas Intimas (2016), con testimonios narrados desde distintas realidades en el mundo con un mismo tema: la violencia doméstica. Su repercusión en la vida de las mujeres desde distintas geografías, la brutalidad masculina, la indefensión, el discurso que se repite en distintas culturas una y otra 
vez, la violencia física contra los cuerpos femeninos. El grito y la denuncia de quienes son violentadas en el espacio más intimo, el discurso contra la misoginia rapaz que oscurece el cotidiano devenir de muchísimas mujeres en el mundo.

Tatiana Huezo, egresada del Centro de Capacitación Cinematográfica con el documental El Lugar más Pequeño (2011), presenta un testimonio de la experiencia de la guerra civil en El Salvador con una bella estética desde la niñez abandonada, nos narra los estragos de la violencia. Son sus principales protagonistas niñas salvadoreñas. Más tarde esta realizadora contará en un relato sobrecogedor con Tempestad (2016), la historia de dos mujeres violentadas desde la corrupción y la impunidad en México con la llamada Guerra contra el Narco. Desde metáforas visuales con un tratamiento del relato y testimonio inicialmente en off y una fuerza de imagen y tono que provoca nuevamente la empatía y el dolor frente a los sucesos de injusticia, de ilegalidad, corrupción y montaje del México en guerra contra la población civil no declarada, pero inmersa en el miedo que atraviesan las protagonistas en sus historias que son nuestras.

\section{CONClusiones}

Estas producciones documentales feministas se perciben como una memoria un retrato cruzado por la violencia, relatos que fueron cambiando en condiciones de producción y recepción de los documentales. Pero desgraciadamente en su mayoría una misma búsqueda está presente a lo largo de más de cien años de historia: la lucha de las mujeres por tener una vida libre de violencia, acoso, machismo, desigualdad. Una búsqueda permanente que promueve la reflexión desde subjetividades individuales pera así modificar las colectivas.

La mirada feminista en el cine empieza también a influir otras narrativas y no necesariamente contadas por mujeres porque la corporeidad no da necesariamente una mirada incluyente y no objetualizada, se puede expresar que los documentales realizados por personas feministas, trasladan un cine realista, e intentan subvertir y representar, el carácter feminista, recrear y reinterpretar una realidad con una intención, una perspectiva de la vida, una necesidad de expresión sobre una realidad que se torna difícil sin la solidaridad y empatía de quienes nos comparten sus historias.

Cuarenta años atrás quienes compartieron la experiencia del colectivo siguen activas desde la docencia y el cine independiente.

Actualmente muchas más jóvenes, algunas egresadas de escuelas de cine o comunicación, desarrollan cada vez más historias que buscan hacer crecer la presencia en las pantallas de las propuestas incluyentes, de diversidades de mundos donde todas y todos podamos tener presencia y representación en las pantallas, pero también acceso a la producción y a la distribución más allá de los grandes monopolios, esto nos permite una equidad de pantallas, sobretodo en Latinoamérica, donde hay enormes esfuerzos para que estas producciones lleguen a mayores audiencias.

Debemos esperar que las ventanas de difusión desde las tecnologías digitales, visibilicen el cine, el documental y el audiovisual hecho por mujeres de todas las miradas posibles. 


\section{Bibliografía}

Amoros, C. (1985). Hacia una crítica de la razón patriarcal. (Vol. 2). Barcelona, España: Anthropos.

Martín, P. T. (2008). Mujeres detrás de cámara. Nuevasociedad (218).

Martín, P. T. (2018). Elena Sánchez Valenzuela. Guadalajara, Jalisco, México: UDG/Cineteca nacional/UNAM.

Mulvey, L. (1975). Placer visual y cine narrativo. Screen (16).

(NFB), N. F. (Productor), Solange Collin, M. L. (Escritor), \& (The Lost Garden: The Life and Cinema of Alice Guy-Blaché (Le jardin oublié: La vie et l'oeuvre d'Alice Guy-Blaché). 1995, C. U. (Dirección). (1996). Le jardin oublié: La vie et l'oeuvre d'Alice Guy-Blaché [Película]. Canadá/Francia.

Sandoval, O. A. (2013). Intervenciones feministas en el cine documental. Conocimientos situados y (auto)representaciones de género en películas de Alina Marazzi y Maricarmen de Lara. Universidad de Granada. Granada: Erasmus Mundus Master's Degree.

\section{Filmografía}

Burkhard, C. (Escritor), \& Burkhard, C. (Dirección). (2008). Trazando Aleida [Película]. México. CCC (Productor), María Inés Roqué, G. M. (Escritor), \& María Inés Roqué, G. M. (Dirección). (1995). Las Compañeras Tienen Grado (1995), [Película]. México.

Colectiva Hilván (Productor), Necoechea, Á. (Escritor), \& Necoechea, Á. (Dirección). (1982). Milagros y Remedios [Película]. México.

Colectivo Cine-Mujer, (Productor) Colectivo (Directoras) Vida de Angél (1982) Película]. México. De Lara, María del Carmen (Productor), Lara, M. d. (Escritor), \& De Lara, María del Carmen M. E. (Dirección). (1986). No les pedimos un viaje a la luna [Película]. México.

Fritz, S. (Escritor), \& Fritz, S. (Dirección). (1984). Yalaltecas [Película]. México.

Gajá, L. (Escritor), \& Gajá, L. (Dirección). (2009). Mi vida adentro [Película]. México-EU.

Gajá, L. (Escritor), \& Gajá, L. (Dirección). (2016). Batallas intimas [Película]. México.

Gustavo Montiel, A. C. (Productor), Roqué, M. I. (Escritor), \& Roqué, M. I. (Dirección). (19952000). Papá Iván [Película]. México Argentina.

Huezo, T. (Escritor), \& Huezo, T. (Dirección). (2011). El Lugar más pequeño [Película]. México.

Huezo, T. (Escritor), \& Huezo, T. (Dirección). (2016). Tempestad [Película]. México.

Miranda, G. (Escritor), \& Miranda, G. (Dirección). (2004). Relatos desde el encierro [Película]. México.

Necoechea, A. (Escritor), \& Necoechea, A. (Dirección). (1985). Bordando la Frontera [Película]. México.

Portillo, L. O. (Escritor), \& Portillo, L. (Dirección). (2001). Señorita extraviada [Película]. México-EU.

UNAM, C. (Productor), Mira, B. (Escritor), \& MIra, B. (Dirección). (1978). Vicios en la Cocina [Película]. México.

UNAM, C. (Productor), Vargas, R. M. (Escritor), \& Vargas, R. M. (Dirección). (1979). Rompiendo el Silencio [Película]. México.

UNAM, C. (Productor), De Lara Rangel y ME Tames, M. d. (Escritor), Tames \& De Lara Rangel, M. d. (Dirección) (1981). No es por gusto [Película]. México.

Vargas, R. M. (Escritor), \& Vargas, R. M. (Dirección). (1978). Cosas de mujeres [Película]. México. 
